\title{
A giant lipoma of the parietal peritoneum: Laparoscopic excision with the parietal peritoneum preserving procedure - a case report with literature review
}

\author{
Hanlim Choi ${ }^{1,2}$, DongHee Ryu ${ }^{1,2^{*}}$ (D) Jae-Woon Choi ${ }^{1,2}$, Yanjie X $\mathrm{U}^{2}$ and Yook Kim ${ }^{3}$
}

\begin{abstract}
Background: Lipomas are very common benign tumors of mature fatty tissue that can occur in any part of the body. However, lipomas of the parietal peritoneum are extremely rare.

Case presentation: A 36-year-old man presented with urinary frequency for 6 months. On computerized tomography of the abdomen and pelvis, a well-defined fatty mass measuring $20 \times 11 \times 6.5 \mathrm{~cm}$ in size, was found in the lower abdominal cavity. We performed a laparoscopic parietal-peritoneum-preserving excision of the mass. The patient was discharged without complications on post-operative day 6.

Conclusions: To our knowledge, a laparoscopic excision with preservation of the parietal peritoneum for a giant parietal peritoneal lipoma has never been reported. Herein, we report a case of a giant lipoma of the parietal peritoneum successfully managed by laparoscopy.
\end{abstract}

Keywords: Giant lipoma, Laparoscopy, Parietal peritoneum, Urinary frequency

\section{Background}

Lipomas are very common benign tumors of mature fatty tissue that can occur in any part of the body. There are a few reports of giant lipoma of mesentery or omentum [1-4]. However, lipomas of the parietal peritoneum are extremely rare. And there have been no reports of extremely large-sized lipomas of the parietal peritoneum. We describe a case of a giant lipoma of the parietal peritoneum causing urinary frequency secondary to external compression of the bladder. This tumor was successfully managed by laparoscopic excision with preservation of the parietal peritoneum.

\footnotetext{
* Correspondence: dhryu@chungbuk.ac.kr

This work was supported by the intramural research grant of Chungbuk National University in 2015.

${ }^{1}$ Department of Surgery, Chungbuk National University Hospital, 776,

1sunhwan-ro Seowon-gu, Cheongju-si Chungcheongbuk-do 28644, South Korea

${ }^{2}$ Department of Surgery, Chungbuk National University College of Medicine, Cheongju, South Korea

Full list of author information is available at the end of the article
}

\section{Case presentation}

A 36-year-old man presented with urinary frequency for 6 months. He had no significant urologic abnormality and no palpable abdominal mass on physical examination. He denied abdominal pain, vomiting, anorexia, or bowel disturbances. There were no specific laboratory abnormalities. The abdomen and pelvis computed tomography scans showed a $20 \times 11 \mathrm{~cm}$, well-defined, fatty mass in the abdominal cavity. A mass was located between the abdominal wall muscles and the peritoneum and compressed bladder (Fig.1a, b). We performed surgery, firstly. The reasons are as follows: (1) the mass was just beneath the abdominal wall, (2) the patient had symptom (urinary frequency), and (3) the mass was considered benign from well-demarcate mass with homogenous features on CT scan. We performed a laparoscopic mass excision with preservation of the parietal peritoneum. Two 11-mm ports were inserted, one supra-umbilically, and the other in the left lower abdomen. A 5-mm port was inserted in the right lower abdomen. A huge, freely mobile, soft mass in the external 


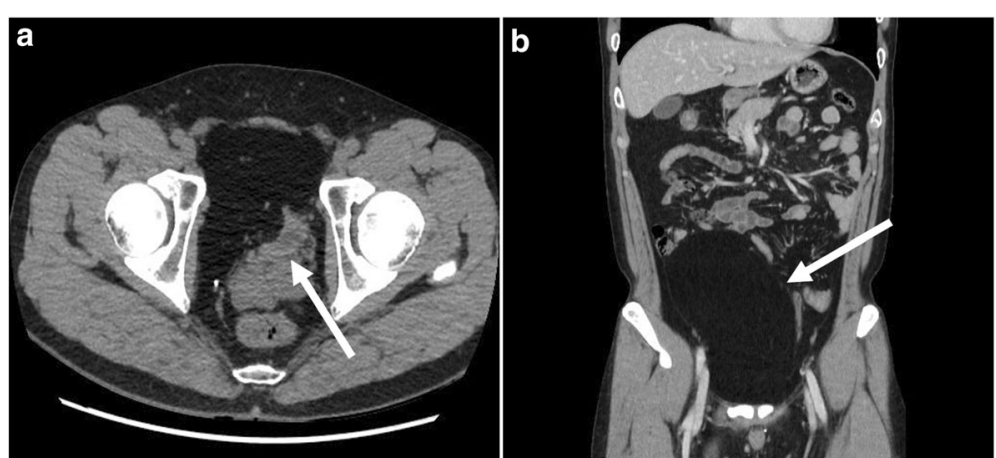

Fig. 1 Contrast-enhanced abdomen-pelvis computed tomography scans. a Axial view. A mass compressed bladder (arrow). b Coronal view. A well-defined homogenous fatty mass measuring $20 \times 11 \mathrm{~cm}$ in size (arrow)

peritoneal layer with no connection to other organs was seen in the lower abdomen (Fig.2a). After demarcating the mass, we excised the parietal peritoneum through the marked line with a monopolar instrument. Next, we dissected the mass from the peritoneum (Fig. 2b). The mass which was excised completely, was placed in a large plastic endopouch-type bag, and extracted through the extended left port site. Finally, the preserved peritoneum was fixed to the abdominal wall using a fixing material with a closed suction drain (Fig. 2c, d). The operative time was $90 \mathrm{~min}$, with no estimated blood loss. The resected specimen size was $22 \times 16 \times 7.5 \mathrm{~cm}^{3}$, and the weight was $942 \mathrm{~g}$. The pathological diagnosis was reported benign lipoma with clear resection margin. The patient was discharged without complications on post-operative day 6 .

\section{Discussion and conclusions}

In 2006, the first case of a lipoma of the parietal peritoneum was reported by Barut et al [5]. Since then, only 5 more cases have been reported (Table 1). In
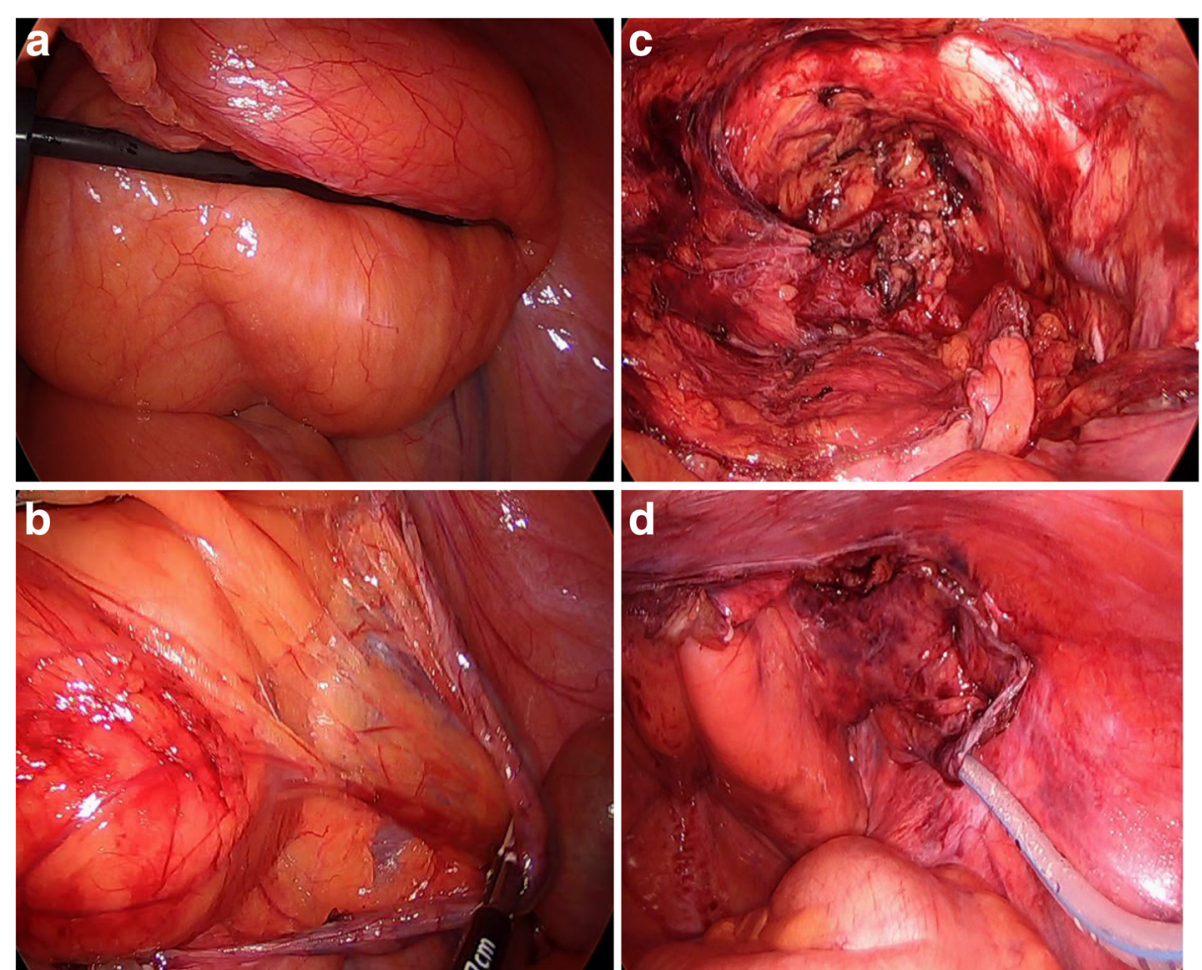

Fig. 2 Laparoscopic view. a A soft huge mass in the external peritoneal layer was seen in the lower abdomen and was free from other organs. b Dissection between mass and peritoneum. c Operative field after mass excision. $\mathbf{d}$ Preserved peritoneum was fixed to abdominal wall using fixation device 
Table 1 Reports regarding the treatment of a lipoma of the parietal peritoneum in the literature

\begin{tabular}{|c|c|c|c|c|c|}
\hline Reference (year) & Age (years) & Sex & Presentation & Surgical procedure & $\begin{array}{l}\text { Maximum } \\
\text { diameter }(\mathrm{cm})\end{array}$ \\
\hline Barut et al. [4] (2006) & 67 & Female & Abd pain, nausea vomiting & Open & 6 \\
\hline Bunker et al. [5] (2013) & 34 & Female & Abd pain & Laparoscopy & - \\
\hline Bang et al. [6] (2014) & 75 & Male & Abd pain, palpable mass & Open & 4.5 \\
\hline Shrestha et al. [7] (2014) & 32 & Male & Abd pain, loss of appetite & Laparoscopy & 3 \\
\hline Sathyakrishna et al. [8] (2014) & 21 & Female & Abd pain & Laparoscopy & - \\
\hline Salgaonkar et al. [9] (2016) & 79 & Male & Abd pain & Laparoscopy & 6.3 \\
\hline Present case (2018) & 36 & Male & Urinary frequency & Laparoscopy & 22 \\
\hline
\end{tabular}

previous reports, all the patients presented with abdominal pain. Three cases were presented with right quadrant abdominal pain mimicking appendicitis, and the largest lipoma had a diameter of $6.3 \mathrm{~cm}$ [6-10]. Our patient presented with urinary frequency caused by external compression of the bladder. A huge and heavy lipoma measuring $22 \times 16 \times 7.5 \mathrm{~cm} 3$ and $942 \mathrm{~g}$ disturbed the filling capacity of the bladder. In 4 previously reported cases, because the lipomas were small in size, they performed a laparoscopic excision of the lipoma and its associated peritoneum [6, 8-10]. In our case, we performed a peritoneal-preserving excision of the lipoma to reduce the pain that we anticipated might be caused by peritoneal resection. Since our patient's lipoma was large in size, we dissected between the peritoneum and the lipoma, and the peritoneum was preserved with fixation around the abdominal wall. The fixation device (Protack ${ }^{\mathrm{T}}$, Medtronic) which is often used in laparoscopic hernioplasties, was useful for fixation. To our knowledge, a laparoscopic excision with preservation of the parietal peritoneum for a giant parietal peritoneal lipoma has never been reported. This procedure is feasible for decreasing postoperative pain and better cosmetic results.

In conclusion, this case highlights the fact that a giant lipoma of the parietal peritoneum can be an unusual cause of urinary disturbances. Laparoscopic procedures are feasible for the excision of a huge lipomas of the parietal peritoneum, and concomitant preservation of the peritoneum is useful for reducing postoperative pain.

\section{Abbreviations}

A-P CT: Abdominal and pelvic computed tomography

\section{Availability of data and materials}

All data generated or analyzed during this study are included in this published article and its supplementary information files.

\section{Authors' contributions}

All authors participated in the management of the patient in this case report. YK assisted in diagnosing field to the patient. DHR and JWC are surgeons, who operated on the patient, supervised and contributed to the case report. $\mathrm{HC}$ and $\mathrm{YX}$ researched and analyzed the data. HC was a major contributor in writing the manuscript, collected all references. All authors read and approved the final manuscript.
Ethics approval and consent to participate

Not applicable.

\section{Consent for publication}

Written informed consent was obtained from the participant for publication of this article and any accompanying tables/images. A copy of the written consent is available for review by the Editor of this journal.

\section{Competing interests}

The authors declare that they have no competing interests.

\section{Publisher's Note}

Springer Nature remains neutral with regard to jurisdictional claims in published maps and institutional affiliations.

\section{Author details}

${ }^{1}$ Department of Surgery, Chungbuk National University Hospital, 776, 1sunhwan-ro Seowon-gu, Cheongju-si Chungcheongbuk-do 28644, South Korea. ${ }^{2}$ Department of Surgery, Chungbuk National University College of Medicine, Cheongju, South Korea. ${ }^{3}$ Department of Radiology, Chungbuk National University Hospital, Cheongju, South Korea.

Received: 9 March 2018 Accepted: 24 July 2018

Published online: 02 August 2018

\section{References}

1. Yang TW, Tsuei YW, Kao CC, Kuo WH, Chen YL, Lin YY. Torsion of a Giant Antimesenteric lipoma of the ileum: a rare cause of acute abdominal pain Am J Case Rep. 2017;18:589-92.

2. Kshirsagar AY, Nangare NR, Gupta V, Vekariya MA, Patankar R, Mahna A, et al. Multiple giant intra abdominal lipomas: A rare presentation. Int J Surg Case Rep. 2014:5(7):399-402.

3. Cha JM, Lee Jl, Joo KR, Choe JW, Jung SW, Shin HP, et al. Giant mesenteric lipoma as an unusual cause of abdominal pain: a case report and a review of the literature. J Korean Med Sci. 2009;24(2):333-6.

4. Shiroshita H, Komori Y, Tajima M, Bandoh T, Arita T, Shiraishi N, Kitano S, et al. Laparoscopic examination and resection for giant lipoma of the omentum: a case report and review of related literature. Surg Laparosc Endosc Percutan Tech. 2009:19(5):e217-e20.

5. Barut I, Tarhan OR, Cerci C, Ciris M, Tasliyar E. Lipoma of parietal peritoneum: an unusual cause of abdominal pain. Ann Saudi Med. 2006;26:388-90.

6. Bunker DL, llie VG, Halder TK. Torsion of an Abdominal-Wall pedunculated lipoma: a rare differential diagnosis for right iliac Fossa pain. Case Rep Surg. 2013;2013:587380. https://doi.org/10.1155/2013/587380.

7. Bang CS, Kim YS, Balik GH, Han SH. A case of lipoma of parietal peritoneum causing abdominal pain. Korean J Gastroenterol. 2014;63:369-72.

8. Shrestha BB, Karmacharya M. Torsion of a lipoma of parietal peritoneum: A rare case mimicking acute appendicitis. J Surg Case Rep. 2014;2014(6). https://doi.org/10.1093/jscr/rju062.

9. Sathyakrishna BR, Boggaram SG, Jannu NR. Twisting lipoma presenting as appendicitis-a rare presentation. J Clin Diagn Res. 2014;8(8):ND07. https:// doi.org/10.7860/JCDR/2014/9663.4728.

10. Salgaonkar HP, Behera RR, Katara AN, Bhandarkar DS. Laparoscopic excision of a lipoma of parietal peritoneum. J Min Access Surg. 2016:12:196-7. 